\title{
H.Pylori Infection Induces CXCL8 Expression and Promotes Gastric Cancer Progress Through Downregulating KLF4
}

zhengxia liu

Anhui Medical University https://orcid.org/0000-0002-9482-3627

Xiao Wu

Anhui Medical University

Yuanyuan Tian

Anhui Medical University

wanchun zhang

Anhui Medical University

siyuan qiao

Anhui Medical University

wenting $\mathrm{xu}$

Anhui Medical University

yakun liu

Anhui Medical University

Siying Wang ( $\sim$ sywang@ahmu.edu.cn )

\section{Research}

Keywords: Helicobacter: pylori (H. pylori), CagA, gastric cancer, CXCL8; Krüppel-like factor 4 (KLF4)

Posted Date: April 20th, 2020

DOl: https://doi.org/10.21203/rs.3.rs-21028/v1

License: (c) (i) This work is licensed under a Creative Commons Attribution 4.0 International License.

Read Full License 


\section{Abstract}

Background Tumour-derived CXCL8 facilitates the movement of myeloid-derived suppressor cells (MDSCs), which have the capacity to restrain antitumour immune responses, into the tumour microenvironment. Kruppel-like factor 4 (KLF4) is a potential tumour suppressor in gastric cancer (GC). However, little is known about the correlation between KLF4 and CXCL8 in gastric carcinoma.

Methods In this study, we used cellular and molecular biological methods to assess whether these two factors interact in GC.

Results We found that the expression levels of CXCL8 and KLF4 were altered in human GC tissues compared to normal gastric tissues in opposite ways. In addition, we found that CagA gene transduction or Helicobacter pylori infection resulted in the upregulation of CXCL8 expression. Knockdown of KLF4 expression increased CXCL8 expression at the protein and RNA levels, whereas overexpression of KLF4 had the opposite effect. Further mechanistic research revealed that KLF4 binds the CXCL8 promoter and negatively regulates CXCL8 at the transcriptional level. Moreover, CXCL8 factor stimulation reduced KLF4 expression, and the CXCL8-mediated increase in GC cell migration and proliferation was reversed by the upregulation of KLF4 expression, eventually promoting neoplasm growth in vivo.

Conclusions Taken together, our findings demonstrate that CXCL8 is a decisive downstream target gene of KLF4 in gastric carcinoma. Targeted KLF4 activation could improve the immunosuppressive microenvironment through direct, negative regulation of CXCL8, providing a new potential target to strengthen the efficacy of immunotherapy in patients with GC.

\section{Background}

Gastric cancer remains a crucial cancer worldwide, as it ranks as the third leading cause of malignant cancer death and the fifth most frequently diagnosed cancer [1]. GC has a poor prognosis due to its high tendency to be advanced, which is the main cause of high cancer mortality [2]. Because the symptoms of $\mathrm{GC}$ are mild and often detected late and due to the limited effectiveness of current treatment options, most people are diagnosed with late-stage GC. In China, in 2015, GC ranked among the top five cancers in terms of mortality and incidence and is thus a significant health problem [3]. The prevalence of H.pylori, a gastric bacterial pathogen aetiologically connected with human gastric carcinoma, correlates with the incidence of GC. H. pylori-associated cytotoxin-associated gene A (CagA) protein, which is transferred to gastric epithelial cells via bacterial type IV secretion, is an oncoprotein that can cause malignant neoplasms in mammals [1, 4]. A large number of studies have shown that $\mathrm{H}$. pylori also promotes the progression, metastasis and recurrence of GC $[5,6]$. This finding may be closely related to the persistence of $\mathrm{H}$. pylori infection, which generates a local immunosuppressive microenvironment, allowing $\mathrm{GC}$ cells to escape immune surveillance [7].

In our previous study, we demonstrated that KLF4 tends to be absent in gastric carcinoma tissue [8]. An increasing number of experimental and clinical research studies have revealed KLF4 to be a tumour- 
suppressor gene in GC [9]. Consistent with the tumour-suppressive function of KLF4, upregulation of KLF4 expression reduces the malignant behaviour of GC cells in vitro [8]. In contrast, CXCL8 acts as a polymorphous chemotactic cytokine to facilitate tumour cell proliferation, migration, and invasion in a paracrine or autocrine manner [10]. CXCL8 is overexpressed in GC and positively associated with poor prognosis and tumour metastasis [11]. In a recent study, CXCL8 was identified as a potent chemotactic factor that promotes the recruitment of myeloid-derived suppressor cells (MDSCs) to tumours through binding CXCR1/2, which was discovered on the surface of tumour-derived MDSCs in a tumour engraftment mouse model. This chemotactic activity can be inhibited in vivo with the use of the CXCR $1 / 2$ blocking agent reparixin [12]. Several recent studies have documented that MDSCs dampen the antitumour immune response mainly by suppressing $T$ cell function via multiple molecular mechanisms [13]. As above, CXCL8 and MDSC interactions play an important role in the tumour immunosuppression microenvironment; thus, inhibiting CXCL8 expression may reduce the recruitment of MDSCs to tumours and improve the tumour microenvironment.

CXCL8 and KLF4 are vital molecules that participate in gastric carcinoma progress, but whether and how the KLF4 and CXCL8 pathways act in a reciprocal manner to manage tumour development are unknown. In the present investigation, we demonstrated for the first time that KLF4 acts as a negative regulator of CXCL8 in GC, providing support for targeted KLF4 activation to improve the immunosuppressive status of tumour patients through suppressing CXCL8 expression and enhancing the effect of comprehensive immunotherapy for GC.

\section{Methods}

\subsection{Cell culture and reagents}

Human normal gastric epithelial (GES-1) cells were cultured in Roswell Park Memorial Institute 1640 medium supplemented with $10 \%$ foetal bovine serum (FBS, Life Technologies/Gibco), $100 \mathrm{U} / \mathrm{mL}$ penicillin, and $100 \mu \mathrm{g} / \mathrm{mL}$ streptomycin (Life Technologies/Gibco), and human GC cell lines (AGS, MGC803) were cultured in Dulbecco's modified Eagle's medium. Cell culture required an environment of $5 \%$ carbon dioxide $\left(\mathrm{CO}_{2}\right)$, and the cells were placed in a humidified $37^{\circ} \mathrm{C}$ incubator. H. pylori was placed in a $37^{\circ} \mathrm{C}$ incubator and cultured in $10 \% \mathrm{CO}_{2}$ and $85 \% \mathrm{~N}_{2}$ environment. Wild-type aSR and CagA plasmids were purchased from Dr Masanori Hatakeyama of Japan's Tokyo University. The antibody against HA was purchased from OriGene, the antibody against KLF4 was purchased from Santa Cruz, the antibody against Ki67 was purchased from Abcam and the antibody against $\beta$-actin was purchased from Proteintech.

\subsection{Reverse transcription-PCR (RT-PCR) and quantitative real-time PCR (qRT-PCR)}

TRIzol reagent (CWBIO, Beijing, China) was used to extract total mRNA from cells. Complementary DNA (cDNA) was reverse transcribed from $2 \mu \mathrm{g}$ of total mRNA with a synthesis kit (Thermo Fisher Scientific, 
Waltham, MA). The following primers (Invitrogen, USA) were used to amplify the CXCL8 gene: forward primer, 5'-AGCCACCGGAGCACTCCATAAG-3'; reverse primer, 5'-CTCCACAACCCTCTGCACCC-3'.

Quantification was carried out using CFX96 Manager software (Bio-Rad). A standard reaction consisted of $2 \mu \mathrm{l}$ of cDNA, $10 \mu \mathrm{l}$ of TaqMan ${ }^{\mathrm{TM}}$ Fast Advanced Master Mix (Applied Biosystems ${ }^{\mathrm{TM}}$ ), $1 \mu \mathrm{l}$ of probe (CXCL8 probe (Hs00174103_m1) or Hprt1 probe (Hs02800695_m1) from Applied BiosystemsTM) and $7 \mu \mathrm{l}$ of RNase-free water. qRT-PCR was carried out with the following standard procedure: $50^{\circ} \mathrm{C}$ for $2 \mathrm{~min}$; $95^{\circ} \mathrm{C}$ for $2 \mathrm{~min}$; and 40 cycles of $95^{\circ} \mathrm{C}$ for $1 \mathrm{~s}, 60^{\circ} \mathrm{C}$ for $20 \mathrm{~s}$, and $72{ }^{\circ} \mathrm{C}$ for $10 \mathrm{~s}$. Data analysis was performed according to the $2^{-\triangle \Delta C T}$ method.

\subsection{ELISA}

Cell culture supernatants were collected by low-speed centrifugation. A human IL-8/CXCL8 Quantikine ELISA kit (R\&D Systems, D8000C) was used to quantify CXCL8 in the cell culture supernatant, heparin plasma, citrate plasma, human serum, and EDTA plasma. Samples were pipetted into the wells of a plate, and the optical density (OD) at $450 \mathrm{~nm}$ was measured using Multiskan Spectrum (Thermo Fisher Scientific).

\subsection{Plasmid construction, transient transfection, and luciferase reporter gene assay}

A CXCL8 promoter DNA fragment containing two KLF4-binding sites was cloned into the pGL3-Basic vector (Promega), and wild-type and mutated reporter plasmids (WT, Site1, Site2, and Double site) were designed (Invitrogen). All of the constructed plasmids were verified by sequencing the inserts and flanking regions. Cells were plated at a density of $4 \times 10^{5}$ cells before transfection with jetPRIME $\circledast$ transfection reagent (Polyplus-transfection SA) according to the manufacturer's protocol. Wild-type or mutant CXCL8 promoter reporter plasmids were transfected into GC cells. Luciferase reporter assays were performed using a dual-luciferase assay kit (Promega, E2940) according to the manufacturer's protocol. Fluorescence intensity was measured with a Multiskan Spectrum (Thermo Fisher Scientific) after transfection.

\subsection{Chromatin immunoprecipitation (ChIP)}

According to the manufacturer's protocol, GC cells $\left(2 \times 10^{6}\right)$ were prepared for ChIP with a ChIP assay kit (Catalogue \# 17-371; Millipore). The resulting precipitated DNA specimens were collected, and fractions of the CXCL8 promoter region were amplified using PCR. The primers designed for PCR are: forward primer, 5'- TGAGGCCAAGGGCCAAGAGA-3'; and reverse primer, 5'- GGCTAGCAGACTAGGGTTGCC - 3'. The products were electrophoretically resolved on a $1.5 \%$ agarose gel and directly viewed using ethidium bromide staining.

\subsection{Western blotting}

Cells were washed twice using cold, sterile phosphate-buffered saline (PBS) and then lysed with RIPA buffer containing $1 \mathrm{mM}$ phenylmethanesulfonyl fluoride (PMSF). Proteins from each sample were loaded 
on an $8 \%$ SDS-polyacrylamide gel and then transferred onto polyvinylidene fluoride (PVDF) membranes (Millipore). Anti-KLF4 antibody and anti- $\beta$-actin antibody were used as primary antibodies.

\subsection{Methyl thiazolyl tetrazolium (MTT), colony-formation, and cell-migration assays}

Cells $(3 \times 103)$ were plated in a 96-well plate after being transfected with the indicated plasmid for $24 \mathrm{~h}$, and an MTT assay was used to measure the cell viability at 0,24 , and $48 \mathrm{~h}$. Five hundred GC cells were plated in a 6-well plate after being treated and grown for 2 weeks. The GC cells $(7 \times 104)$ were placed into Transwell (Corning) membranes for $24 \mathrm{~h}$ after transfection with the indicated plasmid. Then, the cells were washed with PBS, fixed for 15 min, dyed with crystal violet for 30 min, washed with water, and air dried. The cells were counted visually and photographed.

\subsection{Immunohistochemical staining}

To assess the relationship between the protein expression of KLF4 and CXCL8 in GC patients, a tissue microarray (TMA) (HStmA150CS02, Shanghai Outdo Biotech Company) with cancer tissues and corresponding adjacent tissues from 75 pairs of GC samples was used to detect KLF4 and CXCL8 expression. Patient characteristics are summarized in Table 1. In addition, KLF4 and Ki67 protein expression levels in MGC803-control and MGC803-CXCL8 nude mice were detected by immunohistochemistry (IHC). Anti-KLF4 antibody (Santa Cruz, 1:180), anti-CXCL8 antibody (GeneTax, 1:500), and anti-Ki-67 antibody (Abcam, 1:200) were used as primary antibodies. Figures were acquired with MV Image software.

\subsection{Tumour xenograft model}

Two groups of 6 female BALB/c nude mice (4 weeks) each were purchased from the Nanjing Model Animal Center. All mice were maintained in a standard pathogen-free environment on a daily $12 \mathrm{~h}$ light/12 $\mathrm{h}$ dark cycle. MGC803 cells treated with recombinant human CXCL8 protein (named MGC803CXCL8) were expanded and collected in PBS. This was followed by subcutaneous injection of the cells (5 $\times 10^{6} /$ mouse) into the right flanks near the backs of nude mice. Tumour volume (TV) was calculated twice a week according to the formula $0.5 \times$ (width $2 \times$ height). All the mice were euthanized when the tumour reached a size requiring euthanasia based on animal ethics. Tumours were harvested and weighed. Next, tumour tissues were collected for subsequent experiments.

\subsection{Statistical analysis}

All the RT-PCR, Western blot, ELISA and ChIP experiments were conducted in vitro and repeated 2 to 4 times or as indicated. The results of independent experiments are expressed as the mean \pm SEM unless otherwise indicated. Data analysis between groups was carried out with Student's t-test. To analyse correlations between groups, the Pearson chi-square test, Fisher's exact test, or chi-square test was used when appropriate. Statistical analyses were conducted using SPSS 19.0 and GraphPad Prism $6.0\left({ }^{*} \mathrm{P}<\right.$ $0.05, * * \mathrm{P}<0.01, * * * \mathrm{P}<0.001)$. 


\section{Results}

\subsection{The expression levels of KLF4 and CXCL8 are inversely altered in GC tissue}

To explore the correlation between KLF4 and CXCL8 and their clinical significance in GC, we detected CXCL8 and KLF4 expression in a TMA containing seventy-five paired GC tissues and corresponding adjacent non-tumour tissues through IHC. The cancer tissues showed the relatively high expression of CXCL8 and much lower expression of KLF4. In contrast, adjacent non-tumour tissues showed low CXCL8 expression but high KLF4 expression (Fig. 1A, B). Furthermore, using The Cancer Genome Atlas (TCGA) data $(n=440)$, we found a high level of CXCL8 expression in GC samples with $H$. pylori infection $(P=$ 0.003 , Fig. 1C). Taken together, these data strongly support our hypothesis that $H$. pylori infection leads to the upregulation of CXCL8. In addition, CXCL8 expression was positively correlated with tumour grade in stomach adenocarcinoma $(P<0.01$. Figure 1D). Consistently, KLF4 and CXCL8 expression levels were inversely correlated with disease stage, as shown by analysis of GC patient data and human GC tissues (Table 1). These results indicate that the KLF4-CXCL8 signalling pathway plays a crucial role in GC progression and may serve as a notable biomarker for GC treatment.

\subsection{H. pylori infection or CagA transfection increased CXCL8 expression}

Consistent $\mathrm{H}$. pylori infection in GC can induce decreased or silenced KLF4 expression, and the direct transfection of CagA, the main pathogenic factor of $\mathrm{H}$. pylori, into normal gastric mucosal epithelial cells or GC cell lines was shown to cause the significant downregulation of KLF4 expression [14]. To study the relationship between low KLF4 expression in GC and enhanced GC development, we cocultured GES-1 normal gastric mucosal epithelial cells and the AGS/MGC803 GC cell lines with $H$. pylori (The bacteria were divided among the following groups of cells: the control group (without $H$. pylori), 25:1 $(H$. pylori/cell ratio) experimental group, 50:1 experimental group, 100:1 experimental group, and 150:1 experimental group.) or directly transfected the cells with CagA plasmid, following which KLF4 and CXCL8 expression was detected. First, GES-1/AGS/MGC803 cells were cocultured with H. pylori at different concentrations. After 48 h, KLF4 levels had decreased (Supplementary Fig. 1A, B, C), while CXCL8 RNA levels were significantly upregulated (Fig. 2A,B,C Left panel). Cell culture supernatants were collected and used to detect CXCL8 proteins, which showed that $\mathrm{H}$. pylori enhanced CXCL8 expression (Fig. 2A,B,C right panel). To determine whether the pathogenic factor of H. pylori, CagA, would have the same effect, we transfected CagA into the GES-1/AGS/MGC803 cell lines and found that KLF4 expression was downregulated at $48 \mathrm{~h}$ (Supplementary Fig. 1D, E, F), while CXCL8 protein and RNA levels were significantly upregulated (Fig. 2D, E, F), consistent with the effects of $\mathrm{H}$. pylori infection. These results indicate that $\mathrm{H}$. pylori infection can significantly upregulate CXCL8 expression and downregulate KLF4 expression in GC cells. 


\subsection{KLF4 inhibited CXCL8 expression and repressed CXCL8- induced changes in malignant behaviour in GC cells}

In our previous research, we found that $H$. pylori reduced the expression of KLF4, which is contrary to the high expression of CXCL8 observed in GC cells infected with active H. pylori. With this in mind, we studied the interaction between CXCL8 and KLF4 in GC cells in vitro. KLF4 overexpression plasmids were transfected into AGS/MGC803 GC cell lines (Supplementary Fig. 2A, B), and the cells and cell supernatants were collected after treatment for $48 \mathrm{~h}$. CXCL8 expression was significantly downregulated, as shown by qRT-PCR and ELISA (Fig. 3A,B). To further verify the negative regulatory relationship between KLF4 and CXCL8, we transiently transfected siRNA designed against KLF4 (KLF4-siRNA) into GES1 normal gastric mucosal epithelial cells and the gastric cancer cell line AGS with relatively high expression of KLF4 (Supplementary Fig. 2C, D). The results showed that CXCL8 RNA and protein levels were significantly upregulated (Fig. 3C,D), which is contrary to the effects of KLF4 overexpression in GC cells. These findings reveal that CXCL8 may be a downstream gene of KLF4. The results of KLF4 gainand loss-of-function assays explicitly proved that KLF4 plays a significant role in regulating the effects of CXCL8 on GC cells. Increased KLF4 expression remarkably upregulated CXCL8 expression, whereas knockdown of KLF4 expression restrained CXCL8 expression.

CXCL8 promotes changes in malignant behaviour, as shown in a large number of previous experiments $[15,16]$. To investigate whether overexpression of KLF4 could interfere with CXCL8-mediated changes in cell malignant behaviour in GC, we performed MTT, colony-formation and cell-migration assays. CXCL8 significantly enhanced GC cell migration and proliferation compared with those of the control group (Fig. 3E, F, G). When KLF4 overexpression was combined with recombinant CXCL8 protein stimulation, the CXCL8-mediated changes in the malignant behaviour of GC cells were reversed (Fig. 3E, F, G). These results indicate that KLF4 plays an important role in negatively regulating CXCL8-mediated behaviour changes in GC cells.

\subsection{CXCL8 was identified as a downstream target gene of KLF4}

The inverse relationship between KLF4 and CXCL8 expression indicates the transcriptional regulation of CXCL8 expression by KLF4. In fact, bioinformatics analysis showed that the promoter region of CXCL8 contains two potential KLF4-binding sites. We constructed vectors containing wild-type and three sitespecific mutated CXCL8 reporter sequences with a luciferase reporter gene called WT, Site1, Site2, and Double site (Fig. 4A). The inhibitory effect on the CXCL8 promoter was enhanced when the CXCL8 promoter luciferase reporter gene (WT) was cotransfected with a high dose of KLF4 overexpression plasmid into GES-1 cells (Fig. 4B). In AGS cells, as the dose of KLF4 overexpression plasmid increased, KLF4 was found to inhibit CXCL8 promoter luciferase reporter activity (Fig. 4C). The Site1, Site2, and Double site mutation luciferase reporter gene were cotransfected with KLF4 overexpression plasmid into GES-1 and AGS cells. KLF4 had no inhibitory effect on the luciferase activities of cells transfected with the mutated CXCL8 promoter vectors (Fig. 4B, C). CXCL8 promoter mutation analysis indicated that the 
mutated region excluded the response element(s) for the regulation of CXCL8 promoter activity by KLF4. ChIP assays were conducted to examine whether the KLF4 protein could bind the CXCL8 promoter region, which contains two KLF4-binding sites. The results showed that KLF4 protein binding to the CXCL8 promoter region was remarkably increased when the HA-KLF4 expression vector was transfected into AGS and MGC803 cells (Fig. 4D, E). Therefore, KLF4 represses CXCL8 expression, and CXCL8 acts as a crucial downstream target gene of KLF4 in GC.

\subsection{CagA positively regulated the expression of CXCL8 at the transcriptional level}

The critical role of KLF4 in regulating the impact of CXCL8 on GC progression with $H$. pylori infection was further investigated. The CXCL8 promoter reporter activity was significantly enhanced when CagA was used for the cotransfection assay in GES-1 and AGS cells (Fig. 5Ai, 5Aii), which was opposite the effect of KLF4. Then, reciprocal rescue experiments were performed. CagA could reverse the CXCL8-mediated downregulation of KLF4 (Fig. 5Bi, 5Bii), while KLF4 could reverse the CXCL8-mediated upregulation of CagA (Fig. $5 \mathrm{Ci}$, $5 \mathrm{Cii}$ ). These results show that CXCL8 suppression induced by KLF4 could be partially rescued by CagA overexpression, whereas CXCL8 promotion induced by CagA could be partially rescued by KLF4. Combined with a previous study in which CagA suppressed KLF4 expression [8, 14], the mechanism by which CagA positively regulates CXCL8 may function through inhibiting exogenous KLF4 expression.

\subsection{Exogenous CXCL8 reduces KLF4 expression in vitro}

In our previous study, overexpression of KLF4 could inhibit the expression of CXCL8, and H. pyloriinduced GC cells exhibited high CXCL8 expression. Then, we wondered whether CXCL8 would inhibit KLF4 expression in GC cell directly stimulated with the CXCL8 protein. Therefore, recombinant human CXCL8 protein was used to stimulate GES-1/AGS cells over the short term or continuously. Cell pellets were collected after $6 \mathrm{~h}$ and 15 days, and KLF4 expression was detected. The results showed that KLF4 expression decreased regardless of the length of stimulation (Fig. 6A, B). A concentration of $3 \mathrm{ng} / \mathrm{mL}$ was chosen as the proper concentration of recombinant human CXCL8 protein with which to stimulate GES1/AGS cells for different lengths of time to significantly inhibit KLF4 expression. Cells expressing CXCL8 protein that were chronically treated for 15 days were collected to detect the capacity of cells proliferation and migration, showed that CXCL8 protein stimulation promotes GES-1 proliferation, colony formation and migration (Fig. 6C). The same result showed that CXCL8 enhanced AGS cell proliferation and migration (Fig. 6D). These results indicate that CXCL8 negatively regulates KLF4 and promotes cells malignant behaviour change in vitro.

\subsection{CXCL8 promotes GC tumour growth through inhibiting KLF4}


Having found that CXCL8 inversely suppresses KLF4 expression, we established groups of MGC803 cells treated with recombinant human CXCL8 protein for 30 days (named MGC803-CXCL8 cells). Western blot analysis showed that the overexpression of CXCL8 inhibited KLF4 expression in MGC803 cells in vitro (Fig. 7A). Consistently, CXCL8 markedly promoted proliferation and migration in MGC803-CXCL8 cells compared with the control cells (Fig. 6B,C,D). To further investigate whether CXCL8 plays a critical role in promoting tumour growth, the established MGC803-CXCL8 cells mentioned above and MGC803-Control cells were subcutaneously injected into nude mice, after which the tumours were harvested (Fig. 6F). The MGC803-CXCL8 group exhibited accelerated tumour growth compared with the MGC803-Control group in the in vivo assay (Fig. $6 \mathrm{~F}$, right panel). In addition, tumour weight was obviously increased in nude mice injected with MGC803-CXCL8 cells (Fig. 6F, left panel). Moreover, IHC was conducted to analyse the correlation between KLF4 and Ki-67 protein expression in xenograft tumour tissues. Images clearly proved that CXCL8-treated tumour tissue is negatively correlated with KLF4 expression and positively correlated with Ki-67 expression (Fig. 6G). Together, these results indicated that CXCL8 promotes GC tumour growth through inhibiting KLF4 expression.

\section{Discussion}

Studies have shown that transduction of the KLF4 gene into GC tumour cells can significantly increase cell cycle arrest and apoptosis and inhibit tumour growth and metastasis in mice, indicating that KLF4 acts as a tumour-suppressor gene [17]. Additionally, T Yu et al. established the Rosa-Cre+; Klf4fl/fl mouse model in which KLF4 in gastric epithelial cells was deleted by Rosa-Cre and demonstrated that KLF4 deletion enhanced gastric cell proliferation [18]. Many studies support the concept that the suppression of KLF4 expression promotes GC cell proliferation and migration [19-21]. In addition, KLF4 has been recognised as a potential therapeutic target for GC treatment through its attenuation of oncogene function; for example, the KLF4 gene was shown to inhibit STK33 expression by immediately binding the KBS site of its promoter region to control GC progression and metastasis [22]. We found contrasting CXCL8 and KLF4 expression patterns in human GC tissues. KLF4 expression was lower in GC tissue than in adjacent tissue, while the opposite results were observed for CXCL8, indicating a certain link between KLF4 and CXCL8.

CXCL8, the most common inflammatory factor, has a variety of functions in angiogenesis, cell migration, proliferation, and survival [23]. CXCL8 was the most significantly upregulated gene in the whole genome after the infection of normal gastric epithelial cells with $\mathrm{H}$. pylori for $24 \mathrm{~h}$ [24]. CXCL8 is highly expressed in various carcinomas and is associated with poor prognosis [25-28]. In vitro, H. pylori infection or CagA transduction significantly upregulated CXCL8 expression at the RNA and protein levels. These data show that CXCL8 is positively related to $\mathrm{H}$. pylori infection in GC cells. In our previous study, we found that KLF4 is expressed at low levels in $\mathrm{H}$. pylori-positive GC tissue in comparison with $\mathrm{H}$. pylori-negative GC tissue. Moreover, the CagA gene suppresses KLF4 expression and facilitates the proliferation and migration of GC cells in vitro $[8,14]$. These results imply a probable interaction correlation between KLF4 and CXCL8 in $\mathrm{H}$. pylori infection-associated gastric cancer. Then, we investigated the role of the CagA/KLF4/CXCL8 axis in the development of GC. 
Several studies suggest that the combination of CXCR1/2 inhibitors or CXCL8-neutralizing antibodies with other targeted therapies, chemotherapies, and immunotherapy may be more effective in treating cancers than their use alone $[29,30]$. A vast number of studies have investigated the biological actions of CXCL8 with its receptor, CXCR1/CXCR2, and the potential mechanism of CXCL8 in cancer progression [31-33]. However, few studies have examined its signalling pathway at the transcriptional level. Intriguingly, we found that KLF4 overexpression inhibited CXCL8 expression in GC cells, while reduced KLF4 expression upregulated the expression of CXCL8. Behavioural studies showed that GC cell proliferation and migration were enhanced after CXCL8 factor treatment but decreased by KLF4 overexpression. In addition, our previous study showed that CagA could restrict KLF4 through upregulating miR-155 and promoting malignant transformation [8]. Therefore, we hypothesized that CagA upregulates CXCL8 expression through inhibiting KLF4 expression and enhancing the tumour immunosuppressive microenvironment, resisting the effects of immunotherapy. Through mechanistic studies, we have demonstrated for the first time that CXCL8 acts as a downstream target gene of KLF4 and that KLF4 negatively regulates CXCL8 by directly binding its promoter. In addition, we found for the first time that CXCL8 inversely inhibited KLF4 expression, suggesting a signalling pathway between CXCL8 and KLF4. Moreover, we demonstrated that CXCL8 inversely supresses KLF4 expression and promotes tumour growth through in vitro and in vivo assays, implying that a high concentration of CXCL8 could further restrain KLF4 expression in the tumour environment, promote tumour growth, and create an immunosuppressive microenvironment for resistance to the immune response.

Tumours affect bone marrow cell differentiation at different stages, leading to the production of pathologically activated monocytic myeloid-derived suppressor cells (M-MDSCs) and polymorphonuclear myeloid-derived suppressor cells (PMN-MDSCs). MDSCs play a very important role in the tumour immunosuppressive microenvironment [34-37]. A large number of studies have shown that CXCL8 and MDSCs interact in the tumour environment and jointly participate in the formation of a tumour immunosuppressive microenvironment. The expression of CXCL8 and proportion of MDSCs in tumour patients are significantly higher than those in the normal population $[38,39]$. In a recent investigation, MDSCs were found to inhibit T cell proliferation and the production of IFN- $\gamma$ and GrB partly through arginase I production. CXCL8 can induce increased arginase I production in MDSCs via the PI3K-AKT signalling pathway [40]. Given that MDSCs are an important T cell immunosuppressive component in cancer-bearing hosts, the factors that attract these cells to the tumour microenvironment are considered attractive targets for cancer immunotherapy strategies, this is especially true for CXCL8, which is a central component in the recruitment of MDSCs to tumours and has many essential functions in cancer cells $[41,42]$. In recent years, immune checkpoints have emerged as a promising new treatment option in cancer immunotherapy and are currently being developed at a spectacular speed. The targeting of immune checkpoint pathways in GC is under investigation, but the therapeutic effect has not been addressed [43-45]. This may be related to the unique aetiology of GC. Steven et al. reported that CXCR2 is required for the trafficking of MDSCs to the tumour bed and that inhibiting MDSC trafficking to the tumour enhanced the potency of PD1 checkpoint blockade [46]. This work suggests that combining immune checkpoint inhibitors with agents designed to prevent MDSC trafficking to the tumour can 
enhance cancer therapy. Meanwhile, KLF4 is a key regulator that affects GC development and the tumour immunosuppressive microenvironment [47-50]. Combined with our study showing that KLF4 could directly inhibit CXCL8 expression, these results indicate that targeting KLF4 activation to suppress CXCL8 expression may reduce the recruitment of MDSCs to tumours and improve the GC microenvironment.

\section{Conclusions}

In summary, H. pylori or the CagA gene promoted CXCL8 expression, and KLF4 negatively regulated CXCL8 at the transcriptional level. Meanwhile, CXCL8 inversely restrained KLF4 expression and further promoted cell behaviour changes and tumour growth. Our study provides a strong foundation for targeting KLF4 as targeted gene therapy to enhance comprehensive therapy in GC. Further studies are needed to assess the effect of KLF4 on MDSCs and whether KLF4 activation is beneficial to improve the tumour immunosuppressive microenvironment.

\section{Abbreviations}

H.pylori

Helicobacter pylori, KLF4:Krüppel-like factor 4; CagA:Cag pathogenicity island protein A; CXCL8:C-X-C motif chemokine ligand 8; MDSCs:Myeloid-derived suppressor cells;

\section{Declarations}

\section{Availability of data and materials}

The datasets used and/or analysed during the current study are available from the corresponding author on reasonable request.

\section{Funding}

This study was supported by the National Science Foundation of China (NSFC: 81772584).

\section{Competing interests}

The authors declare that they have no conflicts of interest.

\section{Ethics approval and consent to participate}

All animal experiments were performed strictly according to the guidelines of the Institutional Animal Care.

\section{Consent for publication}

Not applicable 


\section{Authors' contributions}

SYW conceived the project and supervised all experiments. ZXL designed the experiments and wrote the manuscript. ZXL and XW analyzed the data. ZXL,XW,YYT,WCZ,SYQ,WTX and YKL provided support with experimental techniques. ZXL extensively modified the manuscript. All authors read and approved the final manuscript.

\section{Acknowledgements}

The authors would like to acknowledge the helpful comments on this paper received from the reviewers.

\section{References}

1. Bray F, Ferlay J, Soerjomataram I, Siegel RL, Torre LA, Jemal A. Global cancer statistics 2018 : GLOBOCAN estimates of incidence and mortality worldwide for 36 cancers in 185 countries. CA Cancer J Clin. 2018;68:394-424.

2. Casamayor M, Morlock R, Maeda H, Ajani J. Targeted literature review of the global burden of gastric cancer. Ecancermedicalscience. 2018;12:883.

3. Chen W, Zheng R, Baade PD, Zhang S, Zeng H, Bray F, Jemal A, Yu XQ, He J. Cancer statistics in China, 2015. CA Cancer J Clin. 2016;66:115-32.

4. Hatakeyama M. Helicobacter pylori CagA and gastric cancer: a paradigm for hit-and-run carcinogenesis. Cell Host Microbe. 2014;15:306-16.

5. Ma HY, Liu XZ, Liang CM. Inflammatory microenvironment contributes to epithelial-mesenchymal transition in gastric cancer. World J Gastroenterol. 2016;22:6619-28.

6. Chongruksut W, Limpakan Yamada S, Chakrabandhu B, Ruengorn C, Nanta S. Correlation of Helicobacter pylori and interleukin-8 mRNA expression in high risk gastric cancer population prediction. World J Gastrointest Oncol. 2016;8:215-21.

7. Lee $\mathrm{K}, \mathrm{Hwang} \mathrm{H}, \mathrm{Nam} \mathrm{KT}$. Immune response and the tumor microenvironment: how they communicate to regulate gastric cancer. Gut Liver. 2014;8:131-9.

8. Ou Y, Ren H, Zhao R, Song L, Liu Z, Xu W, Liu Y, Wang S. Helicobacter pylori CagA promotes the malignant transformation of gastric mucosal epithelial cells through the dysregulation of the miR155/KLF4 signaling pathway. Mol Carcinog. 2019;58:1427-37.

9. Ghaleb AM, Yang VW. Kruppel-like factor 4 (KLF4): What we currently know. Gene. 2017;611:27-37.

10. Alfaro C, Sanmamed MF, Rodriguez-Ruiz ME, Teijeira A, Onate C, Gonzalez A, Ponz M, Schalper KA, Perez-Gracia JL, Melero I. Interleukin-8 in cancer pathogenesis, treatment and follow-up. Cancer Treat Rev. 2017;60:24-31.

11. Lin C, He H, Liu H, Li R, Chen Y, Qi Y, Jiang Q, Chen L, Zhang P, Zhang H, et al. Tumour-associated macrophages-derived CXCL8 determines immune evasion through autonomous PD-L1 expression in gastric cancer. Gut. 2019;68:1764-73. 
12. Alfaro C, Teijeira A, Onate C, Perez G, Sanmamed MF, Andueza MP, Alignani D, Labiano S, Azpilikueta A, Rodriguez-Paulete A, et al. Tumor-Produced Interleukin-8 Attracts Human Myeloid-Derived Suppressor Cells and Elicits Extrusion of Neutrophil Extracellular Traps (NETs). Clin Cancer Res. 2016;22:3924-36.

13. Groth C, Hu X, Weber R, Fleming V, Altevogt P, Utikal J, Umansky V. Immunosuppression mediated by myeloid-derived suppressor cells (MDSCs) during tumour progression. Br J Cancer. 2019;120:16-25.

14. Zhao R, Liu Z, Xu W, Song L, Ren H, Ou Y, Liu Y, Wang S. Helicobacter pylori infection leads to KLF4 inactivation in gastric cancer through a TET1-mediated DNA methylation mechanism. Cancer Med 2020.

15. Khazali AS, Clark AM, Wells A. Inflammatory cytokine IL-8/CXCL8 promotes tumour escape from hepatocyte-induced dormancy. Br J Cancer. 2018;118:566-76.

16. Liubomirski Y, Lerrer S, Meshel T, Morein D, Rubinstein-Achiasaf L, Sprinzak D, Wiemann S, Korner C, Ehrlich M, Ben-Baruch A. Notch-Mediated Tumor-Stroma-Inflammation Networks Promote Invasive Properties and CXCL8 Expression in Triple-Negative Breast Cancer. Front Immunol. 2019;10:804.

17. Wei D, Gong W, Kanai M, Schlunk C, Wang L, Yao JC, Wu TT, Huang S, Xie K. Drastic down-regulation of Kruppel-like factor 4 expression is critical in human gastric cancer development and progression. Cancer Res. 2005;65:2746-54.

18. Yu T, Chen X, Lin T, Liu J, Li M, Zhang W, Xu X, Zhao W, Liu M, Napier DL, et al. KLF4 deletion alters gastric cell lineage and induces MUC2 expression. Cell Death Dis. 2016;7:e2255.

19. Zhao L, Han T, Li Y, Sun J, Zhang S, Liu Y, Shan B, Zheng D, Shi J. The IncRNA SNHG5/miR-32 axis regulates gastric cancer cell proliferation and migration by targeting KLF4. FASEB J. 2017;31:893903.

20. Zheng J, Liu Y, Qiao Y, Zhang L, Lu S. miR-103 Promotes Proliferation and Metastasis by Targeting KLF4 in Gastric Cancer. Int J Mol Sci 2017, 18.

21. Zhang J, Zhu Z, Wu H, Yu Z, Rong Z, Luo Z, Xu Y, Huang K, Qiu Z, Huang C. PODXL, negatively regulated by KLF4, promotes the EMT and metastasis and serves as a novel prognostic indicator of gastric cancer. Gastric Cancer. 2019;22:48-59.

22. Kong F, Sun T, Kong X, Xie D, Li Z, Xie K. Kruppel-like Factor 4 Suppresses Serine/Threonine Kinase 33 Activation and Metastasis of Gastric Cancer through Reversing Epithelial-Mesenchymal Transition. Clin Cancer Res. 2018;24:2440-51.

23. Ha H, Debnath B, Neamati N. Role of the CXCL8-CXCR1/2 Axis in Cancer and Inflammatory Diseases. Theranostics. 2017;7:1543-88.

24. Eftang LL, Esbensen Y, Tannaes TM, Bukholm IR, Bukholm G. Interleukin-8 is the single most upregulated gene in whole genome profiling of $\mathrm{H}$. pylori exposed gastric epithelial cells. BMC Microbiol. 2012;12:9.

25. Li W, Zhang X, Wu F, Zhou Y, Bao Z, Li H, Zheng P, Zhao S. Gastric cancer-derived mesenchymal stromal cells trigger M2 macrophage polarization that promotes metastasis and EMT in gastric cancer. Cell Death Dis. 2019;10:918. 
26. Zhai J, Shen J, Xie G, Wu J, He M, Gao L, Zhang Y, Yao X, Shen L. Cancer-associated fibroblastsderived IL-8 mediates resistance to cisplatin in human gastric cancer. Cancer Lett. 2019;454:37-43.

27. Liu Q, Li A, Yu S, Qin S, Han N, Pestell RG, Han X, Wu K. DACH1 antagonizes CXCL8 to repress tumorigenesis of lung adenocarcinoma and improve prognosis. J Hematol Oncol. 2018;11:53.

28. Song YS, Kim MJ, Sun HJ, Kim HH, Shin HS, Kim YA, Oh BC, Cho SW, Park YJ. Aberrant ThyroidStimulating Hormone Receptor Signaling Increases VEGF-A and CXCL8 Secretion of Thyroid Cancer Cells, Contributing to Angiogenesis and Tumor Growth. Clin Cancer Res. 2019;25:414-25.

29. Viola A, Luster AD. Chemokines and their receptors: drug targets in immunity and inflammation. Annu Rev Pharmacol Toxicol. 2008;48:171-97.

30. David BA, Kubes P. Exploring the complex role of chemokines and chemoattractants in vivo on leukocyte dynamics. Immunol Rev. 2019;289:9-30.

31. Corro C, Healy ME, Engler S, Bodenmiller B, Li Z, Schraml P, Weber A, Frew IJ, Rechsteiner M, Moch H. IL-8 and CXCR1 expression is associated with cancer stem cell-like properties of clear cell renal cancer. J Pathol. 2019;248:377-89.

32. Liotti F, Collina F, Pone E, La Sala L, Franco R, Prevete N, Melillo RM. Interleukin-8, but not the Related Chemokine CXCL1, Sustains an Autocrine Circuit Necessary for the Properties and Functions of Thyroid Cancer Stem Cells. Stem Cells. 2017;35:135-46.

33. Ogawa R, Yamamoto T, Hirai H, Hanada K, Kiyasu Y, Nishikawa G, Mizuno R, Inamoto S, Itatani Y, Sakai Y, Kawada K. Loss of SMAD4 Promotes Colorectal Cancer Progression by Recruiting TumorAssociated Neutrophils via the CXCL1/8-CXCR2 Axis. Clin Cancer Res. 2019;25:2887-99.

34. Wang Y, Ding Y, Guo N, Wang S. MDSCs: Key Criminals of Tumor Pre-metastatic Niche Formation. Front Immunol. 2019;10:172.

35. Gabrilovich DI. Myeloid-Derived Suppressor Cells. Cancer Immunol Res. 2017;5:3-8.

36. Veglia F, Perego M, Gabrilovich D. Myeloid-derived suppressor cells coming of age. Nat Immunol. 2018;19:108-19.

37. Kumar V, Patel S, Tcyganov E, Gabrilovich DI. The Nature of Myeloid-Derived Suppressor Cells in the Tumor Microenvironment. Trends Immunol. 2016;37:208-20.

38. Li P, Chen X, Qin G, Yue D, Zhang Z, Ping Y, Wang D, Zhao X, Song M, Zhao Q, et al. Maelstrom Directs Myeloid-Derived Suppressor Cells to Promote Esophageal Squamous Cell Carcinoma Progression via Activation of the Akt1/RelA/IL8 Signaling Pathway. Cancer Immunol Res. 2018;6:1246-59.

39. Liu Q, Li A, Tian Y, Wu JD, Liu Y, Li T, Chen Y, Han X, Wu K. The CXCL8-CXCR1/2 pathways in cancer. Cytokine Growth Factor Rev. 2016;31:61-71.

40. Mao FY, Zhao YL, Lv YP, Teng YS, Kong H, Liu YG, Wu XL, Hao CJ, Chen W, Duan MB, et al. CD45(+)CD33(low)CD11b(dim) myeloid-derived suppressor cells suppress CD8(+) T cell activity via the IL-6/IL-8-arginase I axis in human gastric cancer. Cell Death Dis. 2018;9:763.

41. Yang L, Wang B, Qin J, Zhou H, Majumdar APN, Peng F. Blockade of CCR5-mediated myeloid derived suppressor cell accumulation enhances anti-PD1 efficacy in gastric cancer. Immunopharmacol 
Immunotoxicol. 2018;40:91-7.

42. Sun L, Wang Q, Chen B, Zhao Y, Shen B, Wang H, Xu J, Zhu M, Zhao X, Xu C, et al. Gastric cancer mesenchymal stem cells derived IL-8 induces PD-L1 expression in gastric cancer cells via STAT3/mTOR-c-Myc signal axis. Cell Death Dis. 2018;9:928.

43. Pico de Coana Y, Choudhury A, Kiessling R. Checkpoint blockade for cancer therapy: revitalizing a suppressed immune system. Trends Mol Med. 2015;21:482-91.

44. Biagioni A, Skalamera I, Peri S, Schiavone N, Cianchi F, Giommoni E, Magnelli L, Papucci L. Update on gastric cancer treatments and gene therapies. Cancer Metastasis Rev. 2019;38:537-48.

45. Salati M, Orsi G, Smyth E, Aprile G, Beretta G, De Vita F, Di Bartolomeo M, Fanotto V, Lonardi S, Morano F, et al. Gastric cancer: Translating novels concepts into clinical practice. Cancer Treat Rev. 2019;79:101889.

46. Highfill SL, Cui Y, Giles AJ, Smith JP, Zhang H, Morse E, Kaplan RN, Mackall CL. Disruption of CXCR2mediated MDSC tumor trafficking enhances anti-PD1 efficacy. Sci Transl Med. 2014;6:237ra267.

47. Leng Z, Li Y, Zhou G, Lv X, Ai W, Li J, Hou L. Kruppel-like factor 4 regulates stemness and mesenchymal properties of colorectal cancer stem cells through the TGF-beta1/Smad/snail pathway. J Cell Mol Med. 2020;24:1866-77.

48. Wang X, Xia S, Li H, Wang X, Li C, Chao Y, Zhang L, Han C. The deubiquitinase USP10 regulates KLF4 stability and suppresses lung tumorigenesis. Cell Death Differ 2019.

49. Wen X, Liu H, Xiao G, Liu X. Downregulation of the transcription factor KLF4 is required for the lineage commitment of T cells. Cell Res. 2011;21:1701-10.

50. Sweet DR, Fan L, Hsieh PN, Jain MK. Kruppel-Like Factors in Vascular Inflammation: Mechanistic Insights and Therapeutic Potential. Front Cardiovasc Med. 2018;5:6.

\section{Figures}


Fig. 1:
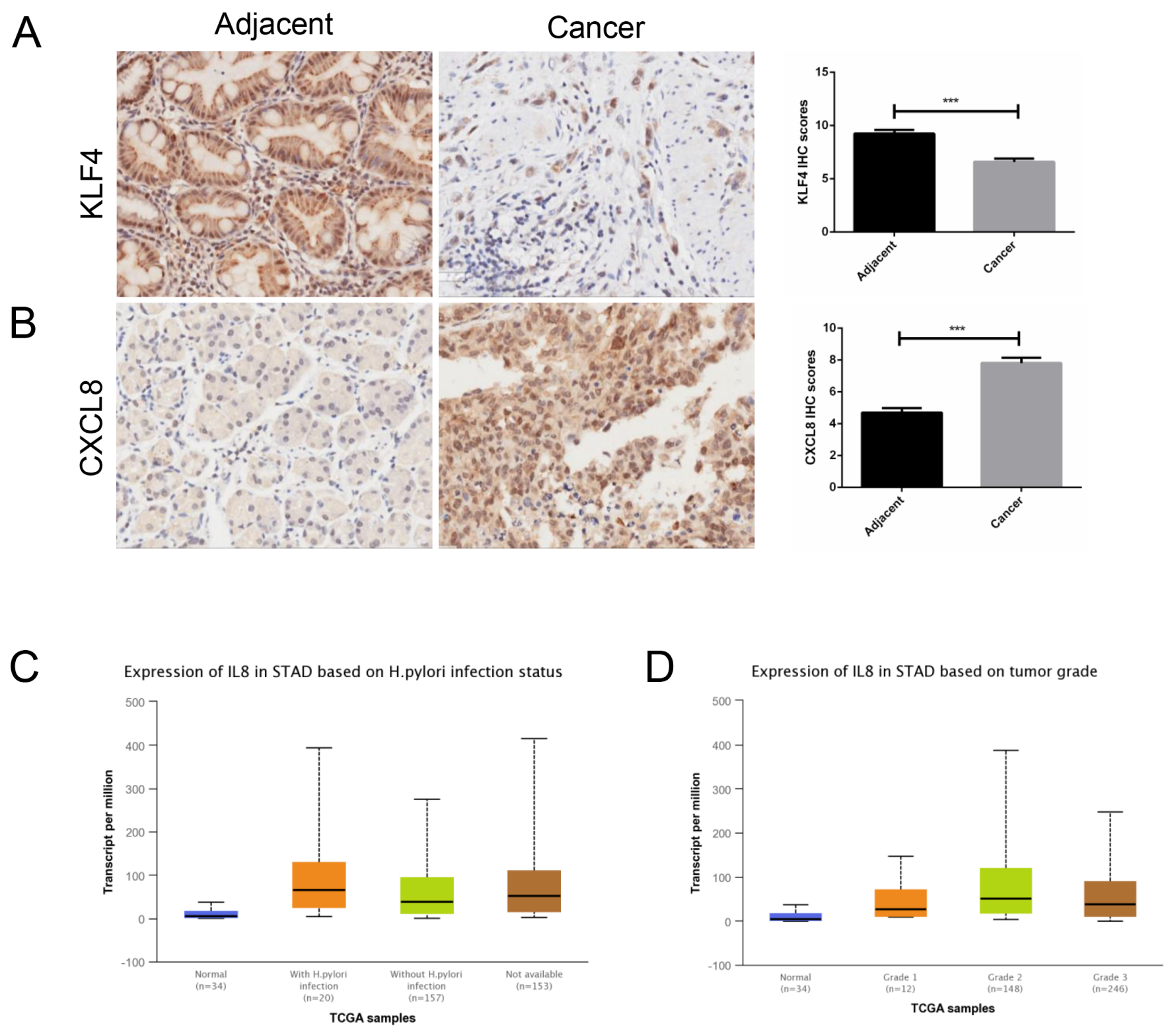

\section{Figure 1}

KLF4 and CXCL8 expression in normal and cancerous gastric tissues. (a) IHC of KLF4 expression in an immunohistochemistry chip (HStmA150CS02) containing gastric cancer tissues and adjacent nontumourous tissues. KLF4 expression was higher in adjacent tissues than in cancer tissues.

Representative images showing KLF4 staining and the average staining scores are given. (b) CXCL8 expression was lower in adjacent tissues than in cancer tissues, and representative images and quantitative graphs of the results of immunohistochemical staining for CXCL8 in GC tissues and corresponding adjacent tissues are shown. (c) CXCL8 expression was higher with $\mathrm{H}$. pylori infection in TCGA data. (d) Increased CXCL8 expression was correlated with a higher tumour grade. 
Fig. 2:

A
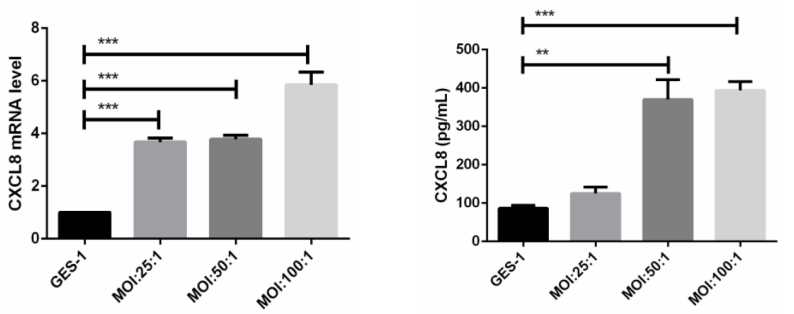

B

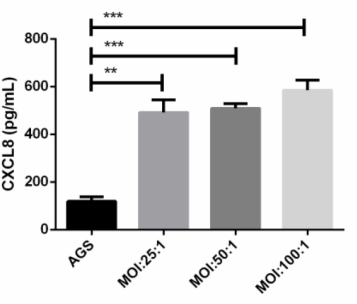

C
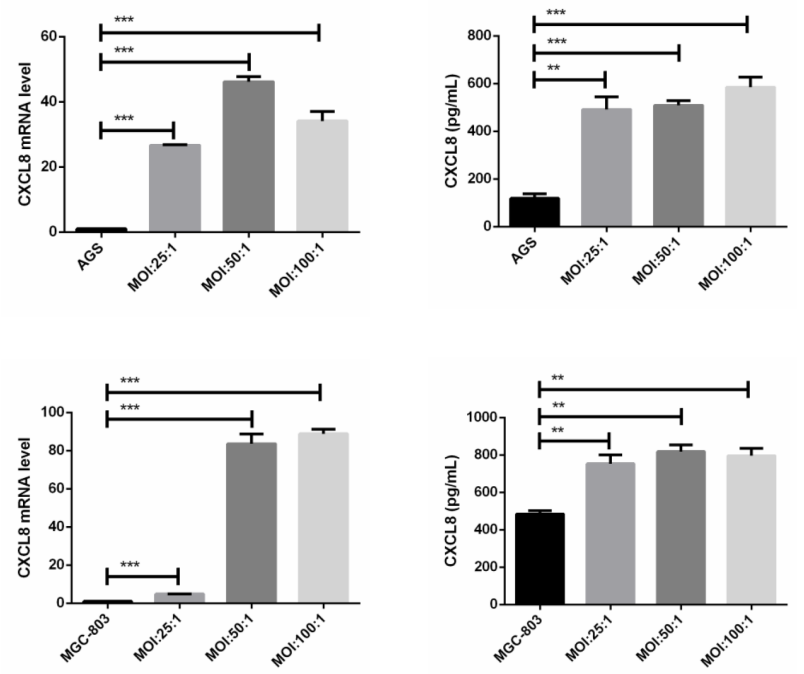

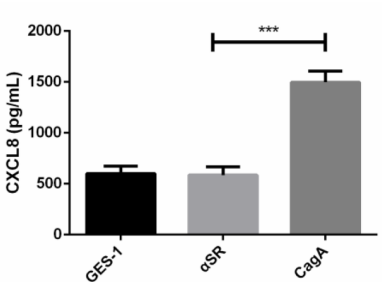

$E$
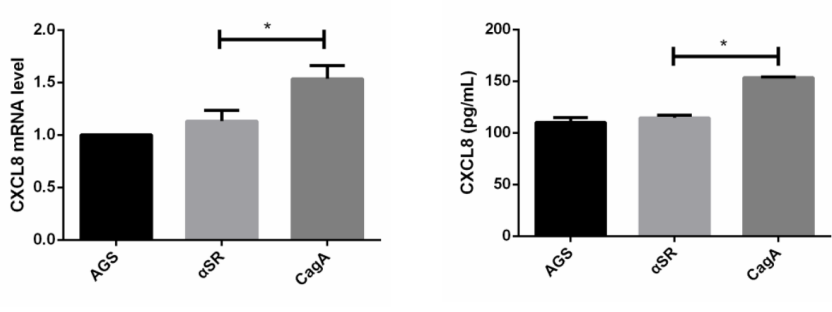

$\mathrm{F}$

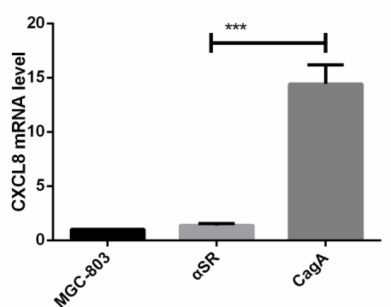

Figure 3

H. pylori infection or CagA transfection induces CXCL8 overexpression. (a) The GES-1 normal gastric mucosal epithelial cell line was cocultured with $\mathrm{H}$. pylori at different concentrations. After 48 h, CXCL8 RNA levels were increased (A, left panel), while CXCL8 protein levels were also upregulated (A, right panel). (b,c) AGS/MGC803 gastric cancer cells were cocultured with $\mathrm{H}$. pylori, and CXCL8 RNA and protein levels were upregulated. (d,e,f) CagA was transfected into the GES-1/AGS/MGC803 cell lines, and after 48 h, CXCL8 protein and RNA levels were significantly upregulated. The experiments were performed independently three times. 
Fig. 3:

A
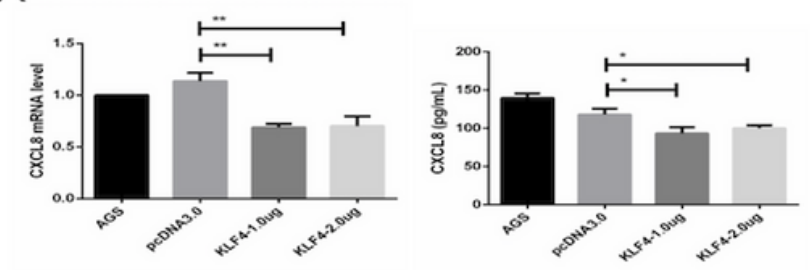

B
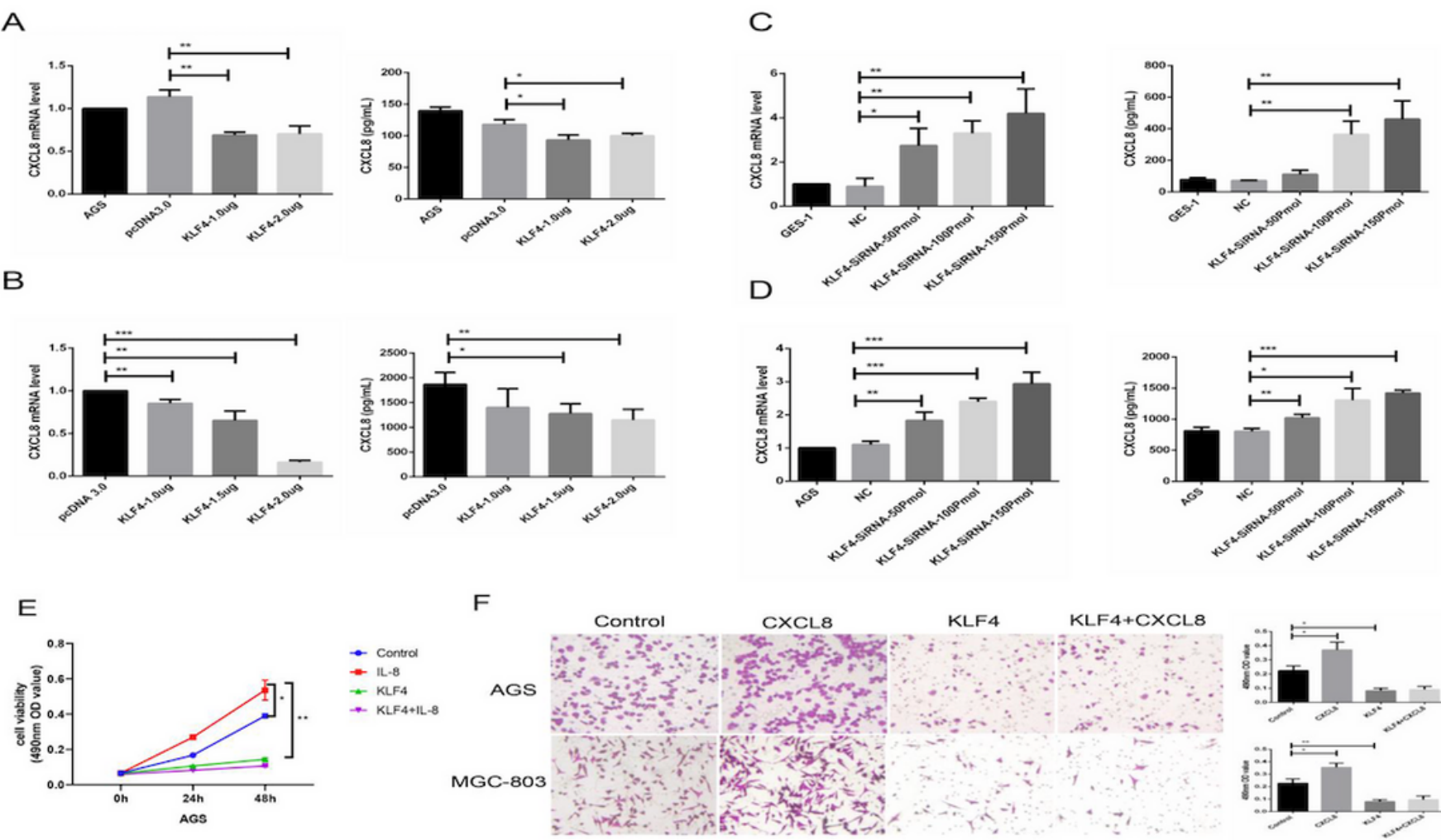

F
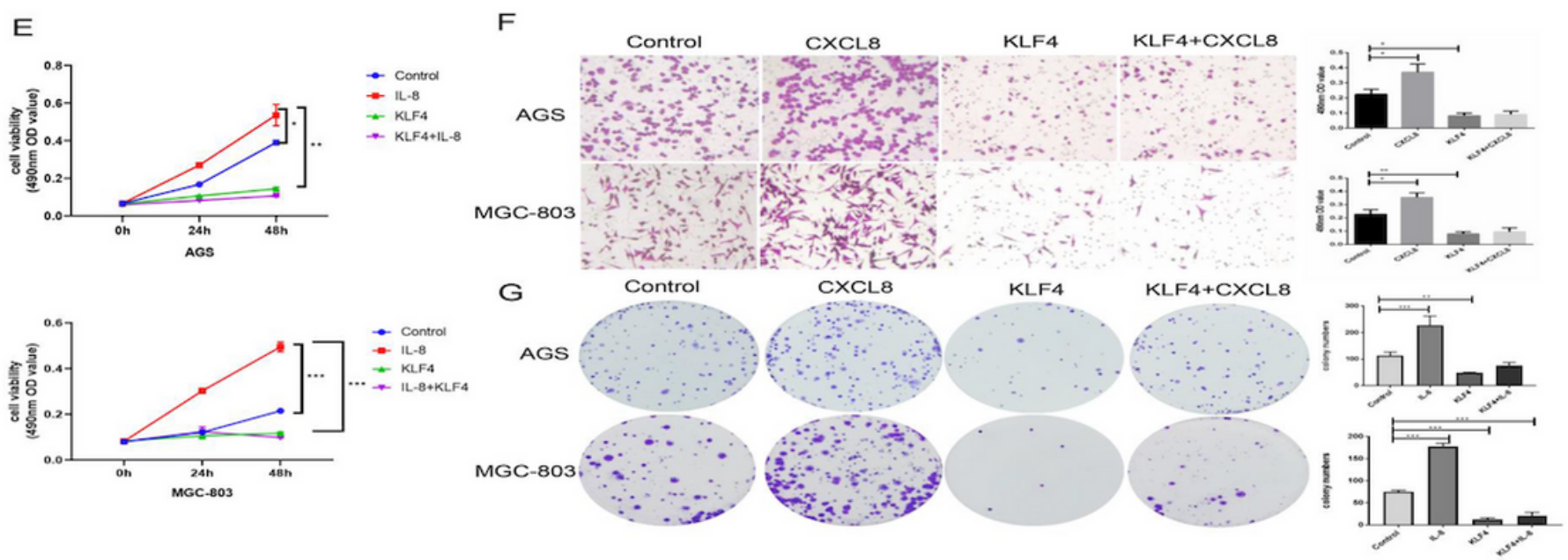

\section{Figure 5}

KLF4 overexpression inhibited CXCL8 expression in gastric cancer cells (a, b) Overexpression of KLF4 in two gastric cancer cell lines and CXCL8 expression were significantly downregulated, as shown by qRTPCR (A,B, left panel) and ELISA (A,B, right panel). (c) KLF4-siRNA was designed and transfected into GES1normal gastric mucosal epithelial cells, after which CXCL8 RNA and protein levels were significantly upregulated. (d) KLF4-siRNA was transfected into the AGS gastric cancer cell line with the relatively high expression of KLF4, and the expression of CXCL8 was significantly upregulated. (e, f, g) Recombinant human CXCL8 protein significantly enhanced gastric cancer cell proliferation (e),migration(f) and colony formation(g), while the CXCL8-mediated increase in gastric cancer cell proliferation and migration was reversed by upregulated KLF4 expression. The experiments were performed independently three times. 
Fig. 4:

A
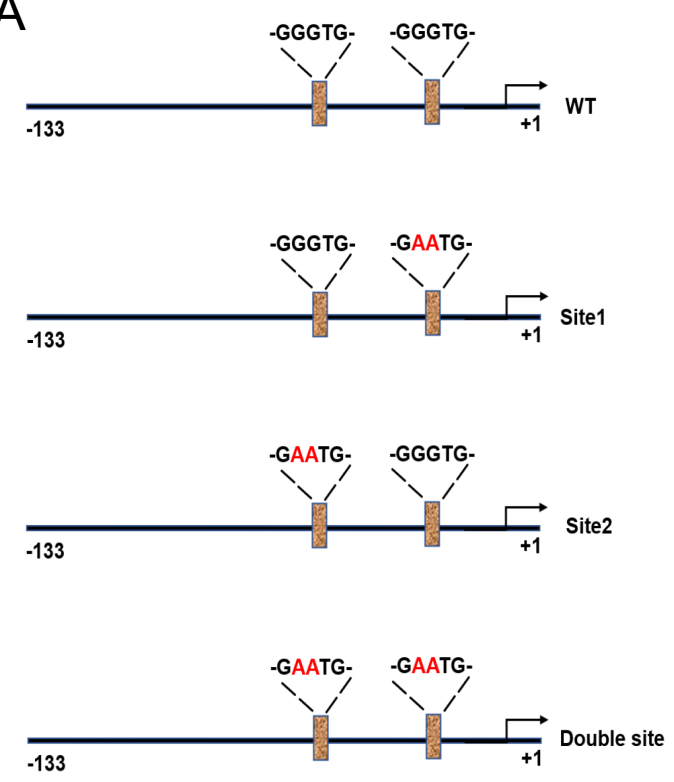

B

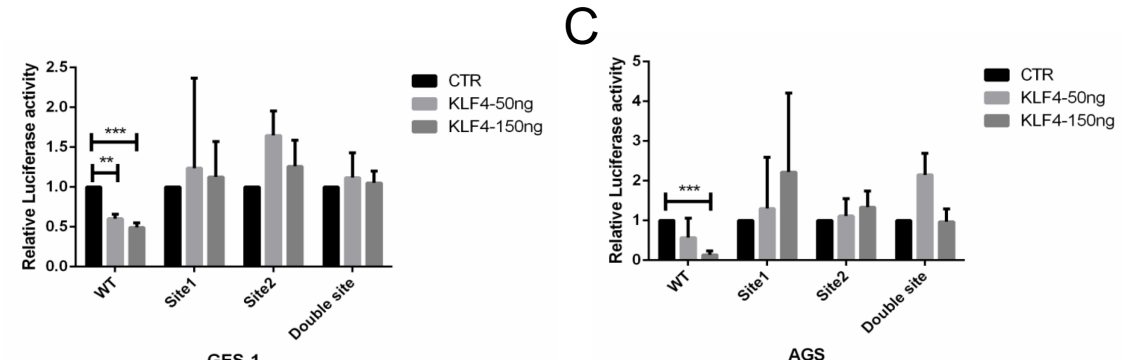

GES-1
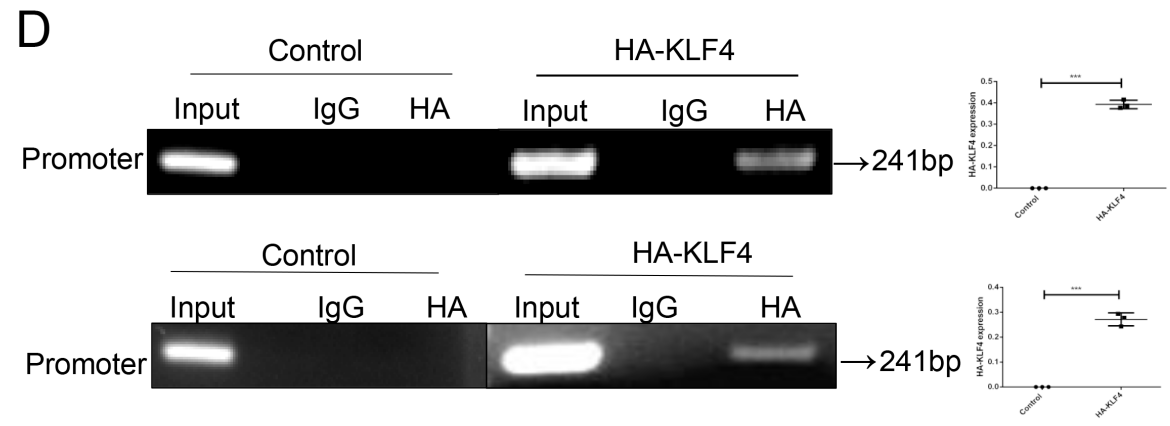

\section{Figure 7}

Negative regulation of CXCL8 expression by KLF4 (a) Schematic of the full-length CXCL8 promoter reporter and its mutant constructs. Two potential KLF4-binding sites are indicated. Vectors containing wild-type and three site-specific mutated CXCL8 promoter sequences and a luciferase reporter gene were constructed and named WT, site1, site2, and double site. (b) The CXCL8 promoter activity in GES-1 cells at $48 \mathrm{~h}$ after transfection with the pcDNA3.1-KLF4 vector is shown. (c) Similarly, the CXCL8 promoter was cotransfected with the pcDNA3.1-KLF4 vector into AGS cells. (d) ChIP analysis of KLF4 binding to the CXCL8 promoter region with a potential KLF4-binding site in AGS gastric cancer cells. The experiments were performed independently three times. 
Fig. 5:

$\mathrm{Ai}$
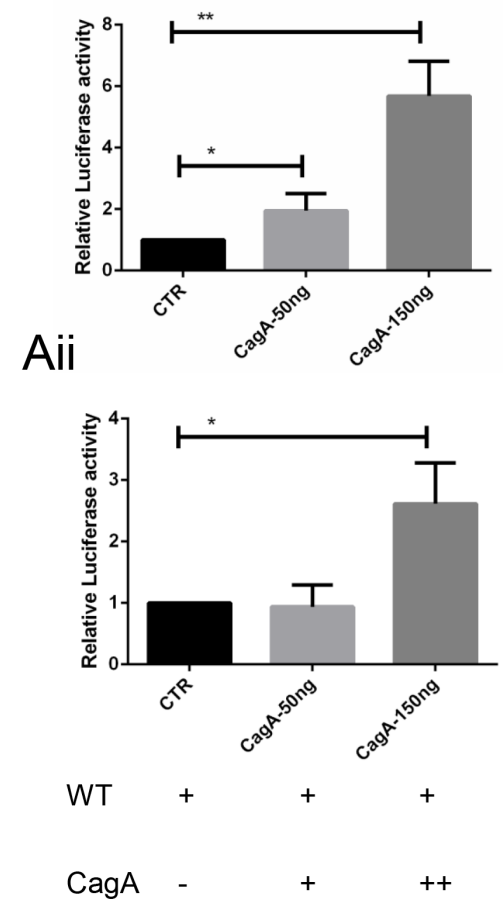

$\mathrm{Bi}$
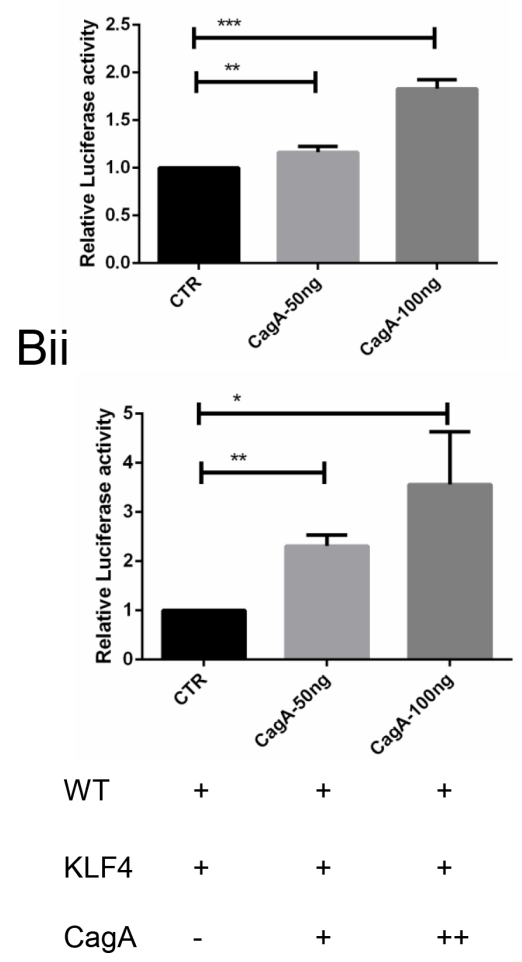

$\mathrm{Ci}$

Cii
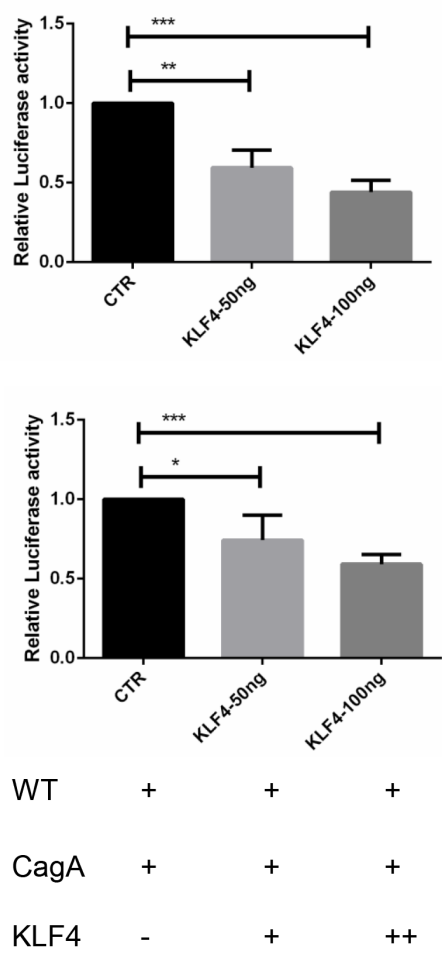

\section{Figure 9}

CagA revised the downregulation of KLF4 on CXCL8 promoter activity (a) CagA co-transfection with the CXCL8 promoter reporter significantly enhanced CXCL8 promoter reporter activity in GES-1 (A, upper) and AGS cells (A, below). (b) CagA can revise the downregulation of KLF4 on CXCL8 in GES-1 (upper) and AGS (below) cells. (c) KLF4 can revise the upregulation of CagA on CXCL8 in GES-1 (upper) and AGS (below) cells. The experiments were performed independently three times. 
Fig. 6:
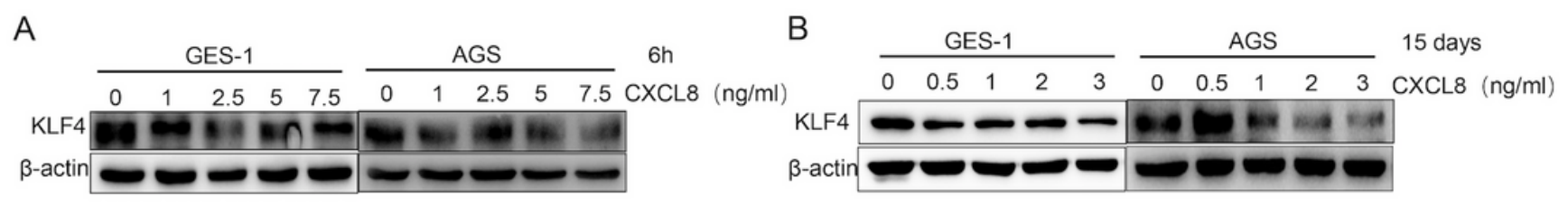

C
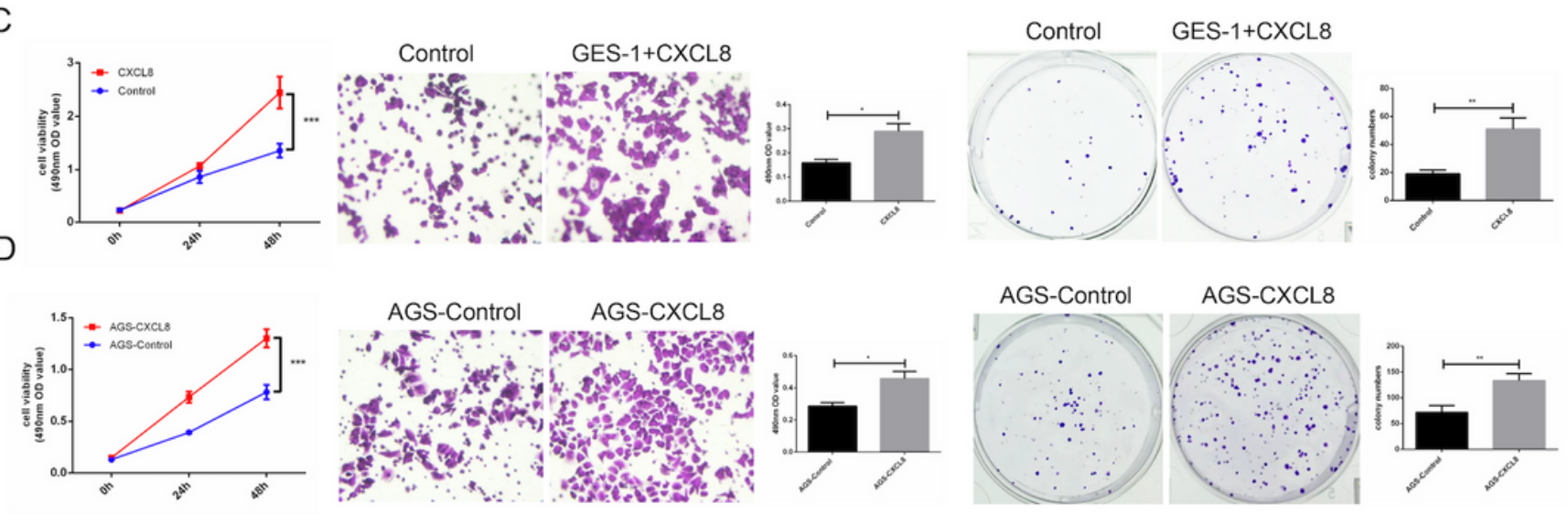

\section{Figure 11}

CXCL8 negatively regulated KLF4 expression and promoted behaviour changes in vitro (a) WB analysis KLF4 expression levels in GES-1 and AGS cells treated with different doses of human CXCL8 recombinant protein for 6 hours. (b) The cells were treated with CXCL8 recombinant protein for 15 days. KLF4 expression decreased as the dose increased. (c, d) The concentration of $3 \mathrm{ng} / \mathrm{mL}$ was chosen to stimulate GES-1 (c) and AGS (d) cells for 15 days, and MTT (left panel), migration (middle panel), colony-formation (right panel) assays were performed. The experiments were performed independently three times. 
Fig. 7:

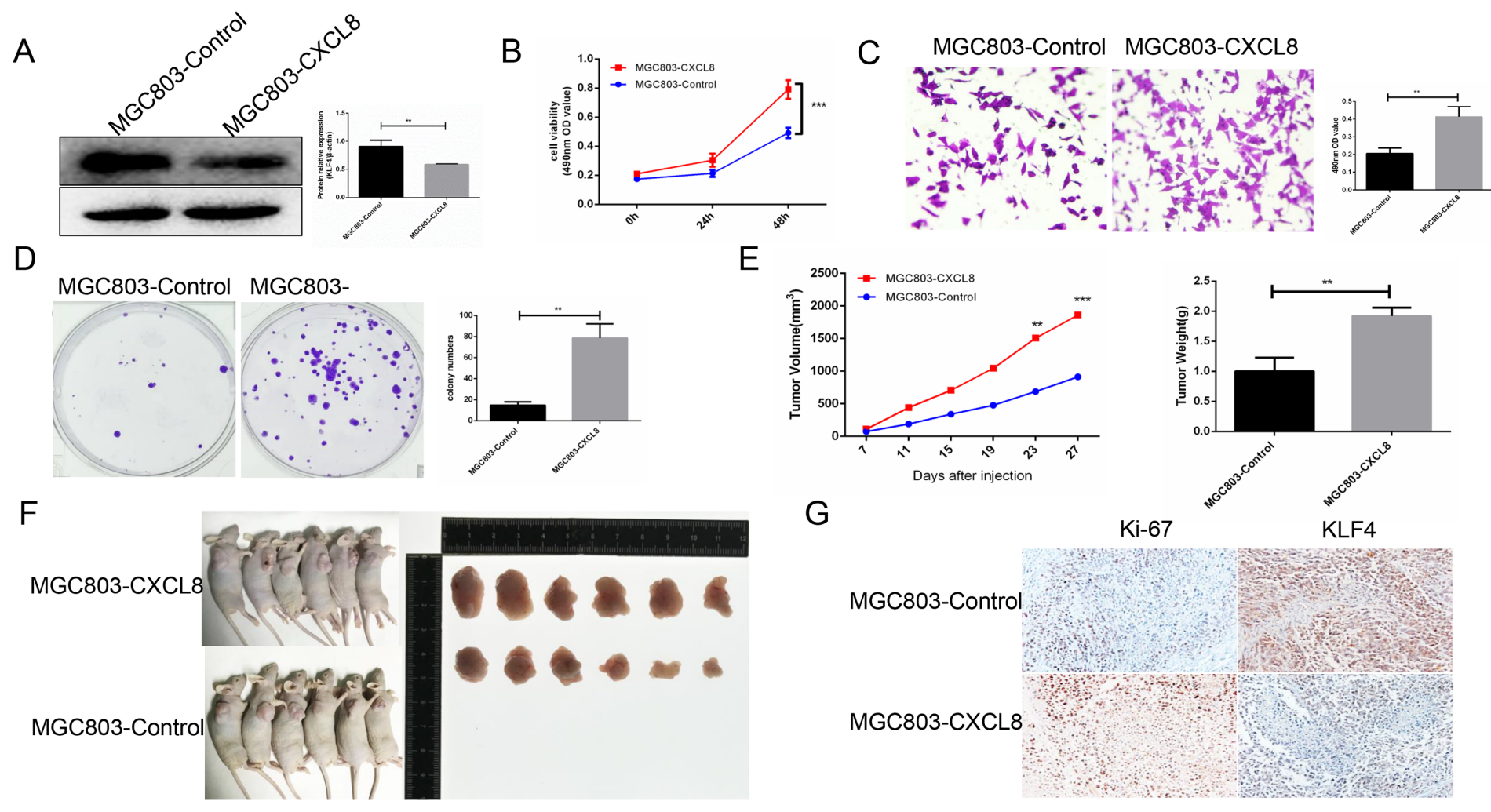

\section{Figure 13}

CXCL8 promotes GC tumour growth through inhibiting KLF4 (a) WB analysis of KLF4 expression levels in MGC803 -CXCL8 cells. (b, c, d) The cells' capacities for proliferation (b), clone formation (d) and migration (c) were significantly increased after CXCL8 treatment for 30 days. (e) Tumour growth curve (left panel) and tumour weight were calculated (right panel). (f) The tumour was removed. (g) IHC analysis of KLF4/Ki67 expression in tumour tissue. The experiments were performed independently three times.

\section{Supplementary Files}

This is a list of supplementary files associated with this preprint. Click to download.

- Supplementaryfigure2.tif

- Supplementaryfigure1.tif

- Coverletter.doc

- Coverletter.doc

- Table1.tif

- Supplementaryfigure2.tif

- Supplementaryfigure1.tif 
- Supplementaryfigure2.tif

- Supplementaryfigure1.tif

- Coverletter.doc

- Table1.tif

- Table1.tif 